\title{
X-ray Structure of Yeast Hal2p, a Major Target of Lithium and Sodium Toxicity, and Identification of Framework Interactions Determining Cation Sensitivity
}

\author{
A. Albert $\dagger^{1,3 *}$, L. Yenush $\dagger^{2}$, M. R. Gil-Mascarell ${ }^{2}$, P. L. Rodriguez ${ }^{2}$ \\ S. Patel ${ }^{3}$, M. Martínez-Ripoll', T. L. Blundell ${ }^{3}$ and R. Serrano ${ }^{2}$
}

${ }^{1}$ Grupo de Cristalografía Macromolecular y Biología Estructural, Instituto de Química Física "Rocasolano" Consejo Superior de Investigaciones Científicas Serrano 119, E-28006 Madrid Spain

${ }^{2}$ Instituto de Biología Molecular y Celular de Plantas

Universidad Politécnica de Valencia-Consejo Superior de Investigaciones Científicas

Camino de Vera s/n

E-46022 Valencia, Spain

${ }^{3}$ Department of Biochemistry University of Cambridge Tennis Court Road, Cambridge CB2 $2 H Q$, UK

${ }^{*}$ Corresponding author
The product of the yeast HAL2 gene (Hal2p) is an in vivo target of sodium and lithium toxicity and its overexpression improves salt tolerance in yeast and plants. Hal2p is a metabolic phosphatase which catalyses the hydrolysis of $3^{\prime}$-phosphoadenosine- 5 '-phosphate (PAP) to AMP. It is, the prototype of an evolutionarily conserved family of PAP phosphatases and the engineering of sodium insensitive enzymes of this group may contribute to the generation of salt-tolerant crops. We have solved the crystal structure of Hal2p in complex with magnesium, lithium and the two products of PAP hydrolysis, AMP and $\mathrm{Pi}$, at $1.6 \AA$ resolution. A functional screening of random mutations of the HAL2 gene in growing yeast generated forms of the enzyme with reduced cation sensitivity. Analysis of these mutants defined a salt bridge (Glu238 ... Arg152) and a hydrophobic bond (Va170 ... Trp293) as important framework interactions determining cation sensitivity. Hal2p belongs to a larger superfamily of lithium-sensitive phosphatases which includes inositol monophosphatase. The hydrophobic interaction mutated in Hal2 $p$ is conserved in this superfamily and its disruption in human inositol monophosphatase also resulted in reduced cation sensitivity.

(C) 2000 Academic Press

Keywords: inositol; 3'-phosphoadenosine-5'-phosphate; protein crystallography; salt tolerance; site-directed mutagenesis

\section{Introduction}

The progressive salinization of irrigated land dictates that an important objective for the agriculture of and regions must be the development of genetically engineered crop plants with greater salt tolerance. Advances in this way will depend on the identification of the primary cellular targets of salt toxicity and elucidation of the underlying mechanisms governing salt responses, which include ion transport, osmotic adjustment and signal transduction (Serrano, 1996). One approach to this complicated problem is the utilization of model organisms, such as Saccharomyces cerevisae.

$\dagger$ A. Albert and L. Yenush made equivalent contributions to the work.

Abbreviations used: PAP, 3'-phosphoadenosine-5'phosphate; IMPase, inositol monophosphatase.

E-mail address of the corresponding author: xalbert@iqfr.csic.es
A striking discovery in this respect was the identification of a major in vivo target of lithium and sodium toxicity, the product of the HAL2 gene (Glaser et al., 1993; Murguia et al., 1995, 1996). Hal2p is a nucleotidase that hydrolyses $3^{\prime}$-phosphoadenosine-5'-phosphate (PAP) to AMP. It requires magnesium for catalysis and it is inhibited by low concentrations of both lithium and sodium. Inhibition of Hal2p during salt stress results in the accumulation of PAP in the cell, which has the potential to produce a variety of toxic effects such as inhibition of sulphotransferases (Roth et al., 1982) and RNA processing enzymes (Dichtl et al., 1997). In yeast, PAP accumulation also blocks the sulphur assimilation pathway (Murguia et al., 1995, 1996).

As previously observed in yeast (Glaser et al., 1993), the overexpression of HAL2 in tomato improves salt tolerance (Arrillaga et al., 1998). This demonstrates the applicability of yeast as a model system for the study of the salinity tolerance of 
higher plants. In addition, a family of Hal2p homologues has recently been identified in plants, including three in Arabidopsis thaliana, AtAHL, AtSAL1, AtSAL2 and one in rice, OsRHL (Peng \& Verma, 1995; Quintero et al., 1996; Gil-Mascarell et al., 1999). These enzymes display varying degrees of cation sensitivity in vitro and studies are underway to examine their ability to improve salt tolerance in plant model systems. Therefore Hal2p is the prototype of an evolutionarily conserved family of PAP phosphatases which may be important determinants of salt toxicity. These enzymes belong to a larger superfamily of phosphatases that exhibit an absolute requirement for divalent metal ions and are inhibited by lithium (York et al., 1995). This superfamily includes fructose-1,6bisphosphate 1-phosphatases (FBPases), inositol polyphosphate 1-phosphatases (IPPases), inositol. monophosphatases (IMPases), PAPases (Hal2p and its homologues) and enzymes acting on both inositol-1,4-bisphosphate and PAP (PIPases) (LopezCoronado et al., 1999). Human IMPase has been most extensively characterized and it has beenproposed as a target for lithium therapy in the treatment of manic depressive patients (Nahorski et al., 1991).

The elucidation of the atomic structure and the manipulation of cation sensitivity of these sodiumsensitive enzymes have potential applications for improving the tolerance of crop plants to $\mathrm{NaCl}$.
Here, we describe the crystal structure of Hal2p complexed with magnesium, lithium, AMP and Pi at $1.6 \AA$ resolution. In addition, as the activity of Hal2p is rate limiting for yeast growth under salt stress, we have identified several residues involved in cation sensitivity using random mutagenesis and in vivo selection of salt-resistant clones. Interestingly, the affected residue in one of the Hal2p mutations has a structural equivalent in human IMPase and the analogous mutation confers lithium tolerance to this enzyme.

\section{Results}

\section{Crystal structure of Hal2p}

The crystal structure of Hal2p (Figure 1(a)) was solved at $1.6 \AA$ by multiple isomorphous replacement techniques using four heavy atom derivatives (Table 1). The enzyme is a monomer of 355 amino acids that can be described as a two-domain structure linked at residue 220 . The $\mathrm{N}$-terminal domain is a member of the $\alpha+\beta$ class and the C-terminal domain is $\alpha / \beta$ with a mixed $\beta$-sheet. The mainly antiparallel $\beta$-sheet of the $\mathrm{N}$-terminal domain includes $\beta$-strands from $\beta 1$ to $\beta 7$ and an $\alpha$ subdomain consisting of helices $\alpha 1$ to $\alpha 4$. The C-terminal domain contains an almost parallel $\beta$-sheet (strands form $\beta 9$ to $\beta 11$ ), sandwiched between two layers of $\alpha$-helices. The active site lies between

Table 1. Data collection, structure solution and refinement statistics

\begin{tabular}{|c|c|c|c|c|c|c|}
\hline Data collection & Native 1 & Native 2 & Lead acetate & $\begin{array}{l}\text { Samarium } \\
\text { acetate }\end{array}$ & $\mathrm{K}_{2} \mathrm{PtCl}_{6}$ & $\mathrm{Gd}_{2}\left(\mathrm{SO}_{4}\right)_{3}$ \\
\hline Space group & $P 2_{1}$ & & & & & \\
\hline Unit cell $\left(\AA_{,}^{\circ}\right)$ & 54.68 & 55.44 & 55.59 & 55.37 & 55.36 & 54.98 \\
\hline$a, b, c$ & $44.91,71.60$ & $45.49,72.35$ & $45.42,72.46$ & $45.66,72.41$ & $45.40,72.13$ & $45.08,71.98$ \\
\hline$\beta$ & 111.08 & 110.71 & 111.36 & 110.70 & 110.74 & 111.96 \\
\hline Resolution $(\AA ̊)$ & 1.6 & 2.7 & 3.2 & 3.0 & 3.0 & 3.5 \\
\hline$I / \sigma(I)$ (ove.11.7-1.6 $\AA$ ) & $8.2 / 1.8$ & & & & & \\
\hline $\begin{array}{l}R_{\text {merge }}(\%) \\
\text { (ove./1.7-1.6 } \AA)\end{array}$ & $7.3 / 39.1$ & & & & & \\
\hline $\begin{array}{l}\text { Completeness }(\%) \\
\text { (ove./1.7-1.6 ̊) }\end{array}$ & $99.7 / 100.0$ & & & & & \\
\hline $\begin{array}{l}\text { Multiplicity } \\
\text { (ove./1.7-1.6 ̊) }\end{array}$ & $5.1 / 3.7$ & & & & & \\
\hline Temp. (K) & 100 & 293 & 293 & 293 & 293 & 293 \\
\hline Number of sites & & & 5 & 7 & 6 & 2 \\
\hline$R_{\text {Cullis }}(c / a)$ & Reference & & $0.54 / 0.51$ & $0.56 / 0.60$ & $0.68 / 0.64$ & $0.75 / 0.84$ \\
\hline Phs. power $(c / a)$ & Reference & & $1.55 / 2.93$ & $1.86 / 2.40$ & $2.55 / 1.90$ & $2.35 / 1.51$ \\
\hline$R_{\text {Cullis }}(c / a)$ & $0.30 / 0.24$ & Reference & $0.62 / 0.59$ & $0.43 / 0.40$ & $0.74 / 0.83$ & \\
\hline Phs. power $(c / a)$ & $4.66 / 7.40$ & Reference & $1.93 / 2.04$ & $3.52 / 4.33$ & $2.20 / 1.35$ & \\
\hline Mean FOM & 0.63 (ref.) & & 0.58 (ref.) & & & \\
\hline \multicolumn{7}{|l|}{ Refinement } \\
\hline Resol. range & \multicolumn{6}{|c|}{$67.4-1.6$} \\
\hline$R_{\text {factor }}($ overall/1.7-1.6 $\AA$ ) & \multicolumn{6}{|c|}{$19.7 / 30.2$} \\
\hline$R_{\text {free }}($ overall/1.7-1.6 $\AA$ ) & \multicolumn{6}{|c|}{$23.5 / 35.1$} \\
\hline Model & \multicolumn{6}{|c|}{$\begin{array}{l}354 \text { amino acids, } 377 \text { water molecules, one AMP molecule, one } \mathrm{PO}_{4} \text { ion, one } \mathrm{SO}_{4} \text { ion, } \\
\text { one beta-mercaptoethanol molecule, } 2 \text { magnesium ions }\end{array}$} \\
\hline Ramachandran & \multicolumn{6}{|c|}{ Only Ser264 in the generously allowed region } \\
\hline Electron density & \multicolumn{6}{|c|}{ Poor quality at residues 27 to 31; no density for residues 1,356 and 357 . } \\
\hline \multicolumn{7}{|c|}{$\begin{array}{l}R_{\text {Merg }}=\Sigma_{h k l} \Sigma i\left|I_{h k l}-I_{h k l}\right| / \Sigma_{h k l} I_{h k l} \\
R_{\text {Cullis }}=\Sigma\left[\left|F_{\mathrm{H}}\right|-\left(\left|F_{\mathrm{PH}}-F_{\mathrm{P}}\right|\right)\right] / \Sigma\left|F_{\mathrm{H}}\right| \text { for centric and acentric reflections }(c / a) \text {. } \\
\text { Phasing power }=\text { mean value of the heavy atom structure amplitudes divided by the residual lack of closure. }\end{array}$} \\
\hline
\end{tabular}


$\mathrm{N}$-terminal and C-terminal domains and is capped by a hairpin or "flap", including residues from 34 to 45 (see Figure 1(a) and (b)).

Crystal structures of inositol monophosphatase (Bone et al., 1992, 1994a,b), inositol polyphosphate 1-phosphatase (York et al., 1994) and fructose-1,6bisphosphatase (Xue et al., 1994; Villeret et al., 1995) have been reported. These enzymes belong to same superfamily as Hal2p (York et al., 1995) but the low sequence homology (less than 20\%) precluded the use of molecular replacement to solve the Hal2p structure. The Hal2p fold is similar to that of these enzymes and the metal sites are structurally conserved at the intersection of topologically equivalent secondary structural elements in a known structures. The main structural differences are in helices $\alpha 3$ and $\alpha 4$, which are not present in other proteins of this superfamily, and in the relative positions of the N-terminal and C-terminal domains.

The crystallization experiments were carried out in the presence of magnesium, lithium and PAP. Two magnesium ions (at sites S1 and S3), a molecule of AMP and a phosphate ion $(\mathrm{Pi})$ can be unequivocally seen in the high resolution electron density map at the active site (Figure 1(c)). Thus, Hal2p has crystallized as a dead end complex with the two reaction products. Mg1 coordinates Glu72 OG1, Asp142 OD1, Ile143 O, a water molecule (W3) and two oxygen atoms of $\mathrm{Pi}$. Mg3 coordinates Glu72 OG2, two oxygen atoms of $\mathrm{Pi}$ and three water molecules. Both metal ions are octahedrally coordinated. A third metal binding site (site 2) is occupied by samarium and platinum when crystals are soaked for preparing heavy atom derivatives. This is known to be the lithium binding site in human inositol monophosphatase (Pollack et al., 1993; Wilkie et al., 1995).

Hal2p phosphatase is very sensitive to lithium, presenting a half-maximal inhibition concentration, $\mathrm{IC}_{50}$, of $0.1 \mathrm{mM}$ (Murguia et al., 1995). Hal2p crystals were grown in the presence of $10 \mathrm{mM}$ lithium, so it is likely that lithium is occupying site 2 in the structure although it cannot be seen by X-ray crystallography since the ion has only two electrons. In the native structure, lithium is expected to be coordinated by AMP OH3', Asp294 OD1, Asp142 OD2 and an oxygen of $\mathrm{Pi}$, with distances and angles in agreement with those of lithium-oxygen complexes found in the Cambridge Crystallographic Database (Allen et al., 1991) and also reported by Glusker (1991). The three metal sites are located in an acidic cavity close to the $C$ termini of $\beta 1$ and $\beta 2$ from the $\mathrm{N}$-terminal domain and the N-terminus of $\alpha 8$ from the C-terminal domain (see Figure 1(b)).

AMP occupies two pockets in the C-terminal domain (Figure 1(d)). The adenosine ring is located between aromatic rings of Tyr288 and His241. The distance and angle between them, around $3.75 \AA$ and 0 degrees, correspond to those often found in aromatic stacking interactions. The $5^{\prime}$ phosphate of AMP occupies a basic pocket, in which Arg281 makes hydrogen bonds to $\mathrm{O} 5,05^{\prime} \mathrm{P}$ and the ribose ring oxygen. This arginine is either conserved or replaced by lysine in plant PAP phosphatases (Gil-Mascarell et al., 1999), pointing to an important role for this salt bridge with the $5^{\prime}$ phosphate of AMP. His241 ND1, Lys267 NZ and Ser264 mainchain $\mathrm{NH}$ are also interacting with $\mathrm{O}^{\prime} \mathrm{P}$. Ser264 and Lys267 are conserved in plant PAPases (GilMascarell et al., 1999). Several water molecules are also occupying the cavity.

The AMP pocket appears to be holding the nucleotide in a high energy conformation with respect to the ground state (about $6 \mathrm{kcal}$ per mol) (CERIUS2, version 3.0, Molecular Simulations). If the PAP O3' ester substrate adopts the same conformation, with an equatorial disposition of the adenosine ring and 5'PO4 with respect to the ribose ring, it would ensure optimal positioning of the ester by pointing towards the metal binding sites.

\section{Functional screening for Hal2p mutants with altered cation sensitivity}

Previous mutagenesis studies with human IMPase have identified several residues affecting lithium sensitivity (Pollack et al., 1993; Gore et al., 1993). Lys36, Glu70, Asp90, Asp93, and Thr95 were shown to be important for catalytic activity, with enzymes mutated at these residues exhibiting less than $0.2 \%$ of wild-type activity. In addition, with the exception of Glu70, mutation of these residues decreased the inhibition of the enzyme by lithium and excess magnesium. This was expected because Asp90 and Asp93 form part of metal site 2 , that is occupied by lithium, while Glu70 only forms part of metal site 1, a site not involved in lithium or magnesium inhibition. The role of Lys36 must be indirect because this residue does not form part of any metal binding site.

All these mutated residues are conserved in the superfamily of lithium-sensitive phosphatases, corresponding to Lys33, Glu72, Asp142, Asp145 and Thr147 in Hal2p. However, the dual participation of these amino acids in catalysis and metal binding make them of little value for altering the cation sensitivity of active enzymes and therefore for understanding the molecular basis of the differences in cation sensitivity exhibited by different members of the Hal2p and RVIPase enzyme family.

We hypothesized that perturbation of some framework interactions within the enzymes could result in subtle modifications of the active site, preserving catalytic activity but altering sensitivity to inhibitory cations bound at metal site 2 . However, it was very difficult to predict from the Hal2p or IMPase structures what kind of interactions determine the cation affinity and specificity of metal site 2. Three residues of human IMPase, His217, Cys218 and Trp219 could be mutagenized to produce active enzymes less sensitive to lithium and magnesium inhibition (Pollack et al., 1993; Gore et al., 1993; Rees-Milton et al., 1997). His217 

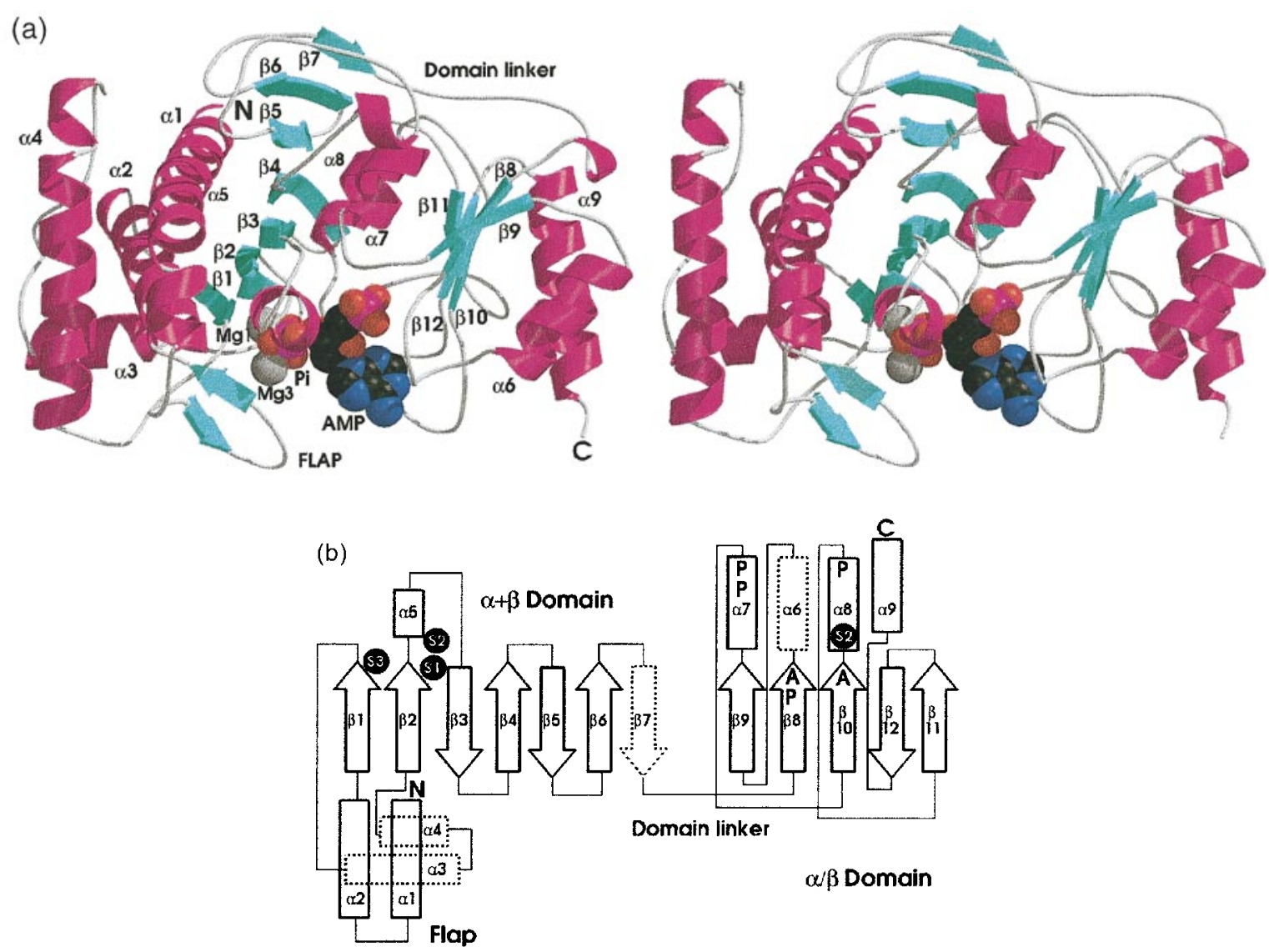

(c)
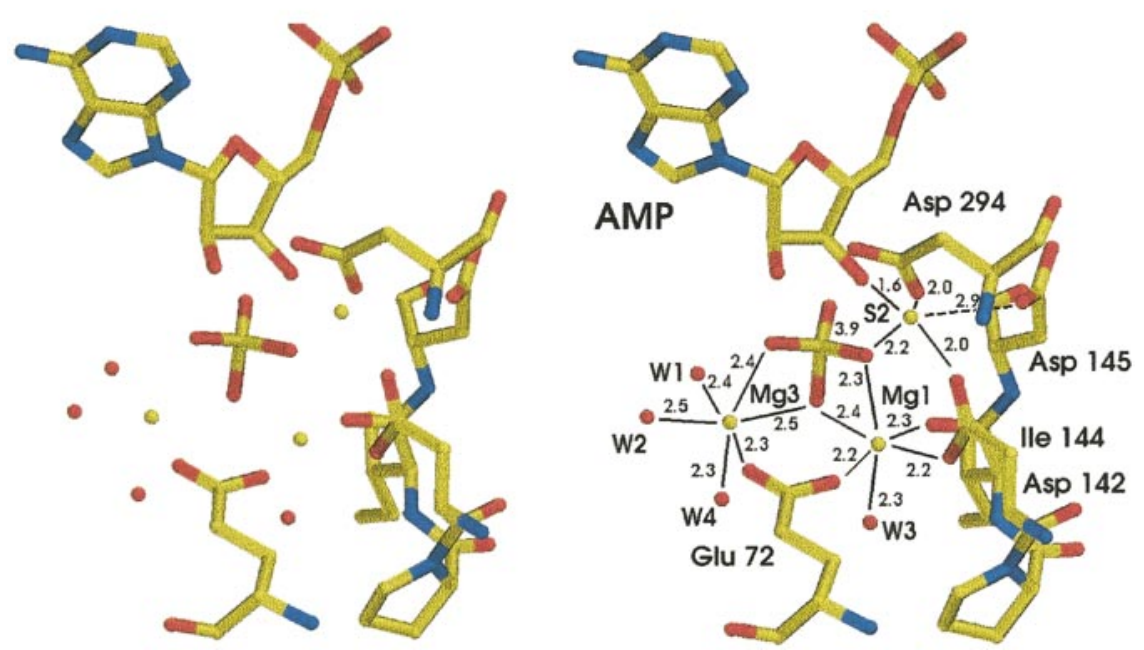

Figure 1 (legend opposite)

and Cys218 are not conserved in the family of lithium-sensitive phosphatases and no structural basis for the effects of these mutations were provided.

One important advantage of the yeast Hal2p system is that this enzyme is rate limiting for growth under lithium or sodium stress (Glaser et al., 1993; Murguia et al., 1995). This means that a functional screening can be made in vivo for ran- dom mutations in Hal2p that result in an active enzyme exhibiting reduced cation sensitivity. As a first step in our analysis of the structural determinants for lithium and sodium inhibition in Hal2p and other phosphatases of the same family, we have generated a library of random mutations in the HAL2 gene and selected for those enzymes conferring lithium resistance to a yeast strain lacking the native HAL2 gene. Of the approximately 


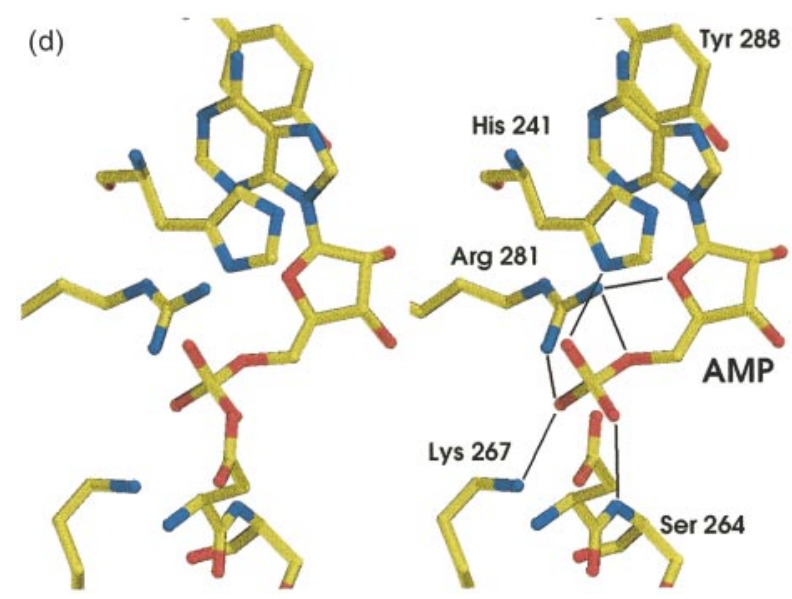

Figure 1. (a) Ribbon representation of Hal2p structure. Magnesium atoms are displayed in gray, AMP and Pi are displayed in CPK mode. (b) Schematic representation of Hal2p showing all the secondary structural elements and binding sites. Continuous lines and broken lines represent conserved or non-conserved secondary structural elements among the sugar enzymes structural class; A is adenosine ring binding site and P is AMP, P5' binding site. (c) Active site in Hal2p. A view of the metal binding sites (distances are in $\AA$ ). (d) PAP binding site.

600,000 potential mutations screened, three clones were identified that upon retransformation into yeast improved growth in the presence of $100 \mathrm{mM}$ lithium chloride (Figure 2(a)). Sequence analysis revealed that two of the three clones contained an identical single point mutation resulting in the substitution of Glu238 to Lys (E238K). The third clone contained a distinct point mutation that gives a Val to Ala substitution at amino acid 70 (V70A).

As the PAP-agarose purification protocol used for native Hal2p depends, in part, on the catalytic inhibition by lithium (see Materials and Methods), the genes encoding the wild-type Hal2p and the mutations E238K and V70A were expressed in Escherichia coli and purified as histidine-tagged fusion proteins. The in vitro characterization of the kinetic properties of the enzymes is summarized in Table 2. The two mutations display increasing levels of lithium and sodium resistance (Figure 2(b) and (c)) while maintaining a relatively high specific activity, as expected from the observation that both proteins complement the absence of Hal2p in yeast (Figure 2(a) and data not shown). As determined by HPLC analysis, the $K_{\mathrm{m}}$ value for the PAP substrate was also altered in the mutant enzymes: the E238 K mutation lowered the affinity for the substrate by fivefold, giving a $K_{\mathrm{m}}$ value of $20 \mu \mathrm{M}$ as compared to $4 \mu \mathrm{M}$ for the wild-type Hal2p. By contrast, the V70A mutation displays an even greater substrate affinity. The $K_{\mathrm{m}}$ value for this mutation of Hal2p was below the limits of detection of the BPLC analysis, indicating that the $K_{\mathrm{m}}$ value is less than $1 \mu \mathrm{M}$ (data not shown).

\section{Construction of an analogous mutation in human inositol monophosphatase}

Analysis of the three-dimensional structures of Hal2p and inositol monophosphatases indicated that Val70, but not Glu238, has a structural equivalent in human IMPase (Ile68). We hypothesized that Ile68 of human IMPase, by making an hydrophobic contact with Trp219, may play an analogous role in defining the cation sensitivity of this enzyme. Thus, using a similar approach to that used for Hal $2 p$ mutants, we prepared histidinetagged fusion proteins of both the wild-type and mutant (I6SA) human IMPase. As observed for the V70A mutation in Hal2p, the I68A mutation in human IMPase is much less sensitive to inhibition by lithium chloride $\left(\mathrm{IC}_{50} 5 \mathrm{mM}\right.$, as compared to $0.6 \mathrm{mM}$ for the wild-type), demonstrating that the change in the active site induced by this amino acid substitution (I68A) results in an equivalent change in the lithium sensitivity of human IMPase (Figure 3).

\section{Discussion}

The structure of Hal2p described here provides the first characterization at the atomic level of both a PAP phosphatase and a physiological target of sodium toxicity (Glaser et al., 1993; Murgia et al., 1995, 1996; Serrano, 1996; Gil-Mascarell et al., 1999).

The crystal structure of Hal2p is similar to the intermediate state just after hydrolysis of the ester, with the two products of the reaction, AMP and $\mathrm{Pi}$, trapped in a dead-end lithium complex (Figure 1(c)). This is consistent with the fact that, in the presence of lithium, Hal2p binds to the immobilized PAP, thus facilitating the purification and the re-utilization of the affinity column. If this step is performed without lithium, Hal2p extensively hydrolyses the immobilized PAP of the column and affinity purification is much less efficient. These results would support that lithium may act as an uncompetitive inhibitor as in the case of 

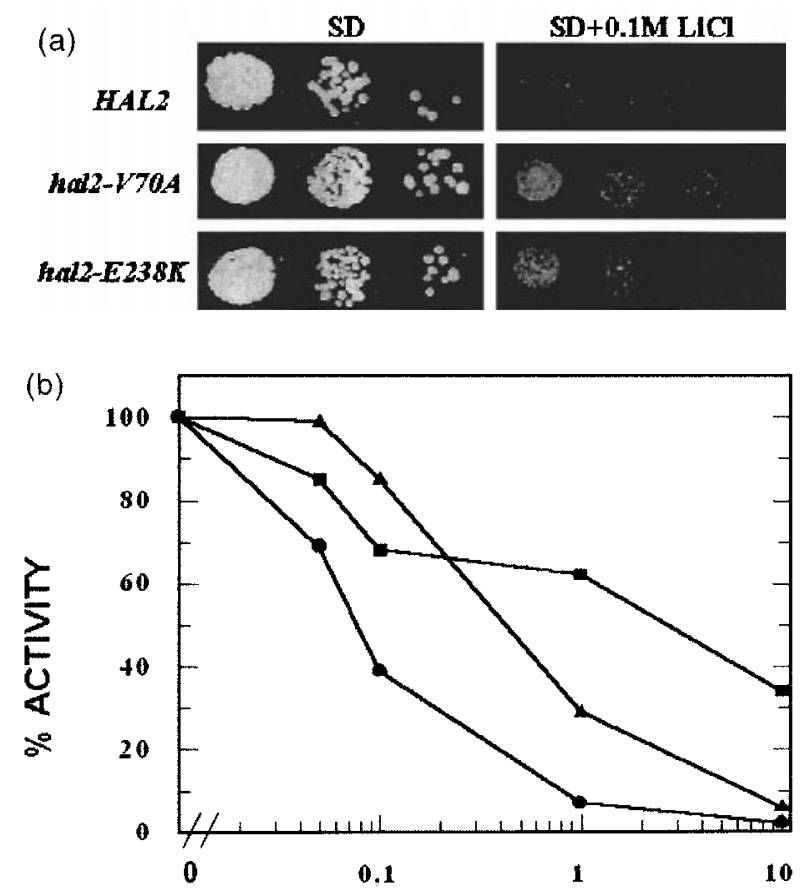

[LiCl], mM

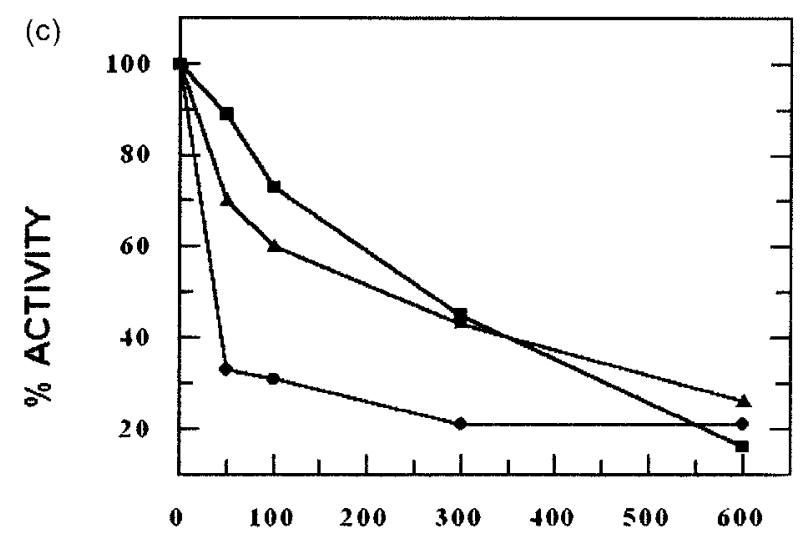

[NaCl], $\mathrm{mM}$

Figure 2. (a) Comparison of the growth of a Hal2yeast strain expressing the wild-type and mutant hal2. Saturated cultures of the three strains were serially diluted and plated on minimal media (SD), with and without lithium chloride $(0.1 \mathrm{M})$. Relative inhibition of phosphatase activity of Hal2p and mutations by lithium chloride (b) and sodium chloride (c). Symbols are as follows: Hal2p (circles); V70A (triangles); E238K (squares). Enzymes were produced as histidine-tagged fusion proteins and incubated with increasing amounts of lithium or sodium. Activity was assayed by the malachite green method, as described in Materials and Methods. Data are expressed as the percentage of activity in the absence of inhibitory cations and are representative of at least two independent experiments performed in duplicate.

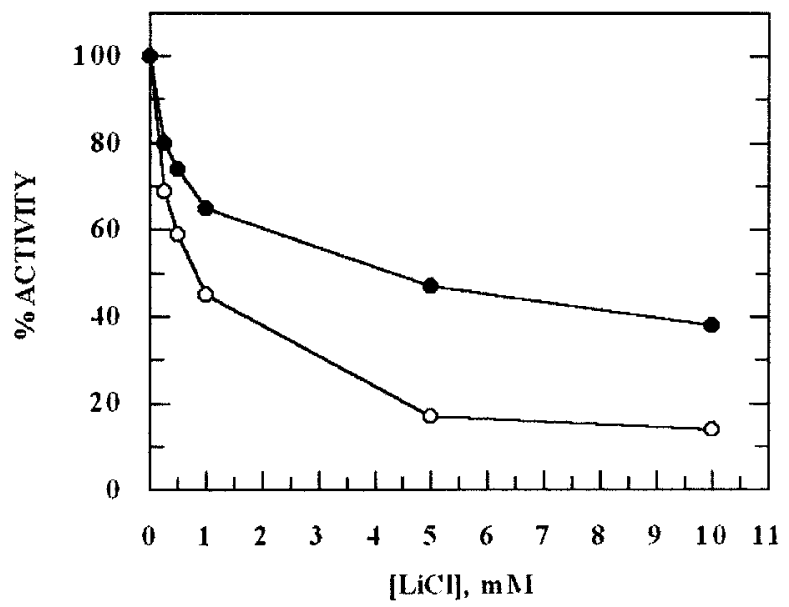

Figure 3. Relative inhibition of phosphatase activity of wild-type (open circles) and mutant (I68A) (filled circles) human IMP by lithium chloride. Experimental procedure is described in Figure 2. Data are representative of two independent experiments performed in duplicate and are expressed as the percentage of activity in the absence of lithium chloride.

other lithium sensitive phosphatases (Nahorski et al., 1991), which means that the inhibition is substrate-enhanced.

We assume that a magnesium ion occupies site 2 and we model Pi bonded to the sugar ring at $\mathrm{O}^{\prime}$. Then, Asp294 OD1 and a water molecule would complete the octahedral coordination at this site as in the platinum derivative (see Figure 1(c)). Following Cole et al. (1995), in this situation, this water would be close enough to the ester bond to be the nucleophile required for hydrolysis. Once the ester is broken, the leaving Pi would move towards magnesium site 3 . The magnesium at site 2 would have fewer ligands and would leave the enzyme together with the AMP and followed by $\mathrm{Pi}$ and magnesium site 3 . Alternatively, W3 at site 1, would act as the nucleophile (York et al., 1994; Bone et al., 1994a,b; Villeret et al., 1995; Strater et al., 1996). As discussed by Wilkie et al. (1995), the final test for these alternative models will come when the stereochemical course of the reaction with respect to the phosphorous atom is determined.

One important difference between the binding of PAP to Hal2p and that of inositol phosphates (Bone et al., 1992, 1994a,b; York et al., 1994) and fructose-1,6-bisphosphate (Xue et al., 1994; Villeret et al., 1995) to their specific enzymes is an aromatic stacking interaction of the adenine ring (Figure 1(d)). Of the two residues involved, Tyr288 is conserved in plant PAP phosphatases, while His241 is conserved in Arabidopsis SAL1 but not in the other two members of the Arabidopsis family (SAL2 and AHL). Interestingly, SAL1 has the best affinity for PAP of all the Arabidopsis PAP phosphatases (Gil-Mascarell et al., 1999). Inositol-1,4bisphosphate resembles the ribose- $3^{\prime}, 5^{\prime}$-bisphosphate moiety of PAP and it is hydrolysed by 
Table 2. Summary of the kinetic analysis of Hal2p mutants

\begin{tabular}{lcccc}
\hline & $K_{\mathrm{m}}(\mu \mathrm{M})$ & $K_{\text {cat }}(1 / \mathrm{seg})$ & $\mathrm{IC}_{50} \mathrm{LiCl}(\mathrm{mM})$ & $\mathrm{IC} 50 \mathrm{NaCl}(\mathrm{mM})$ \\
\hline Hal2p & 4 & 1.7 & 0.07 & 20 \\
E238 K & 20 & 1.7 & 4 & 265 \\
V70A & $<1$ & 0.7 & 0.5 & 200 \\
\hline
\end{tabular}

Hal2p. However, the $K_{\mathrm{m}}$ of this inositol derivative is very high (about $1 \mathrm{mM}$ ), while PAP is hydrolysed with much better affinity $(3 \mu \mathrm{M})$ (data not shown). The absence of aromatic stacking with inositol-1,4-bisphosphate may explain the poor affinity of Hal2p for this substrate.

Hal2p (Murguia et al., 1995) and other plant PAP phosphatases (SL1, SAL2 and AHL1) (Quintero et al., 1996; Gil-Mascarell et al., 1999) are sensitive to sodium and previous work demonstrates that sodium inhibits in the same way as lithium (Gil-Mascarell et al., 1999). Interestingly, yeast IMPases are sodium-sensitive (Lopez et al., 1999) while animal enzymes acting on inositol phosphates and PAP are sodium insensitive (LopezCoronado et al., 1999). It is tempting to speculate that, given the crucial role of these enzymes in cellular physiology, the evolution of animal cells in sodium-containing media (physiological saline) produced sodium-insensitive enzymes. On the other hand, yeast and plants evolved in sodiumpoor media and most of their lithium-sensitive phosphatases are also inhibited by sodium. Only in relatively recent times, when the progressive salinization of irrigation land in semi-arid regions has exposed plants to high sodium concentrations, has this "Achilles tendon" of non-animal cells been uncovered (Serrano, 1996).

A detailed comparison of the structure of sodium-resistant animal enzymes with the structure of Hal2p provides some clues for the structural basis of sodium inhibition. A salt bridge between Arg152 and Asp263 in Hal2p forces a displacement of helix $\alpha 5$ (residues from 147 to 152) with respect to its position in sodium-resistant enzymes. In this situation Hal2p Asp145 OD2 is able to make hydrogen bonds to Asp142 OD2, Asp294 OD2 and Gln265 NE1 (Figure 4(a)). These interactions fix the carboxylate side-chain in a conformation that makes the distance from site 2 to Asp145 OD2 larger than in human inositol monophosphatase (Figure 5). There are two direct consequences of this change. First, lithium is no longer coordinated by Asp145; it is replaced in the coordination sphere by $\mathrm{O}^{\prime}$ from AMP. Second, this enlargement may enable the formation of a sodium complex that blocks the product AMP in the active site. This salt bridge is not present in the sodiumresistant mammalian enzymes. Arg152 is fully conserved in plant PAPases, while Glu238 is conserved in the most sodium-sensitive of these enzymes, AHL (Gil-Mascarell et al., 1999).

In addition to the structural determinants of sodium-lithium discrimination discussed above, framework interactions affecting inhibition by both lithium and sodium may also be important to engineer sodium-tolerant PAP phosphatases. The characterization of the E238 $\mathrm{K}$ mutant reveals an additional role of the salt bridge discussed above in modulating the sodium and lithium sensitivity in Hal2p (Table 2 and Figure 2(b) and (c)). In the wild-type enzyme, the ionic pair between Arg152 and Asp263 is further stabilized by an ionic network involving two adjacent carboxylates Glu154 and Glu238 (Figure 4(b)). It is known that this kind of ion network is essential for increasing the stability and hence, thermoresistance in various enzymes (Goldman, 1995). The mutation E238 K likely changes the stability and the geometry of the salt bridge, the position of $\alpha 5$ and, hence, the sensitivity to lithium and sodium ions. Further evidence of the role of the ionic network can be found by analysing the $\mathrm{IC}_{50}$ values for lithium and sodium ions of the PAP phosphatases of Arabidopsis. The SAL2 gene product conserves Arg152, Asp263 and Glu154, but Glu238 is replaced by His and thus mimics the E238K mutation of Hal2p. As predicted by the hypothesis, the $\mathrm{IC}_{50}$ for both lithium and sodium are very similar for SAL2 and the E238K mutant (10 and $200 \mathrm{mM}$, respectively). Furthermore, the ionic network is conserved in SAL1 gene product, but Glu154 is replaced by Asp. Despite this conservative change, with respect to wild-type Hal2p, this enzyme is very sensitive to lithium but moderately sensitive to sodium ions $\left(\mathrm{IC}_{50}\right.$ for $\mathrm{Li}^{+}$ and $\mathrm{Na}^{+} 0.2$ and $200 \mathrm{mM}$, respectively). This phenotype may be explained by a decrease in the physical size of metal binding site 2, which would prevent the larger sodium ions from binding. In the AHL gene product, Asp263 is replaced by Tyr, thus the equivalent salt bridge is not conserved. However, it would be possible to form a salt bridge involving Arg152 and Glu238. This enzyme is highly sensitive to sodium, but not lithium $\left(\mathrm{IC}_{50}\right.$ for $\mathrm{Li}^{+}$and $\mathrm{Na}^{+} 10$ and $50 \mathrm{mM}$, respectively (GilMascarell et al., 1999)), possibly suggesting an increase in the size of metal binding site 2, such that the smaller lithium ion is no longer stably coordinated. This natural variability in amino acid sequence and cation sensitivity would imply that small changes in the size and/or geometry of site 2 , as defined in part by this ionic network, modulate the cation affinity of this site.

Additional structural elements determining cation sensitivity have been revealed by the V70A mutation. This mutation is also resistant to both sodium and lithium inhibition, but the structural mechanism of this change in sensitivity is difficult 
(a)

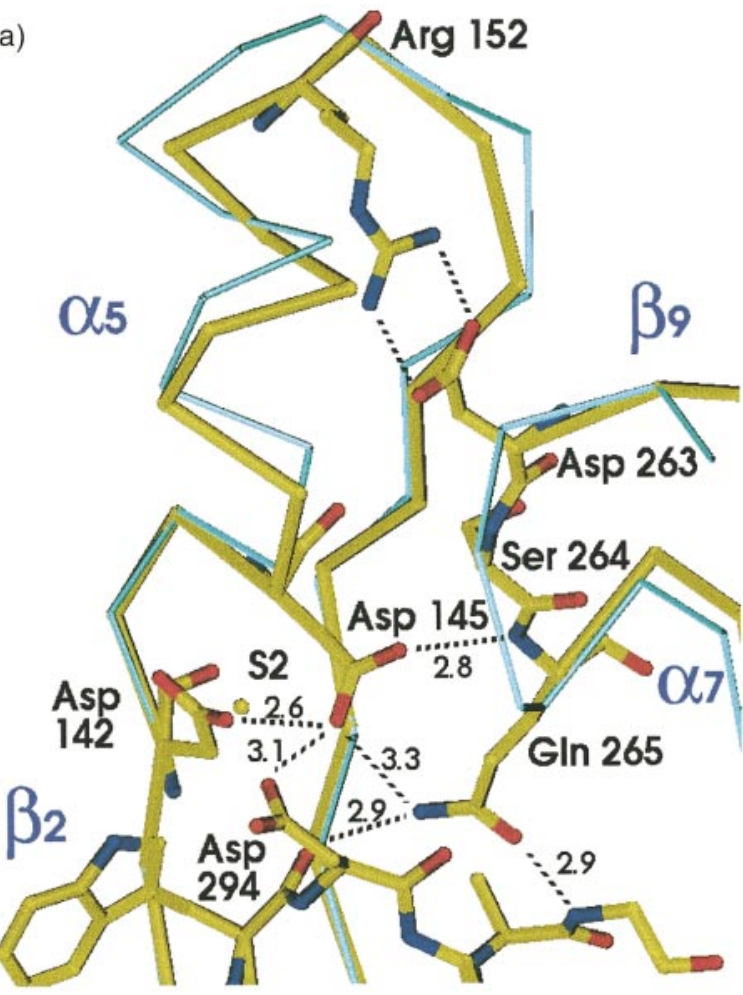

(b)

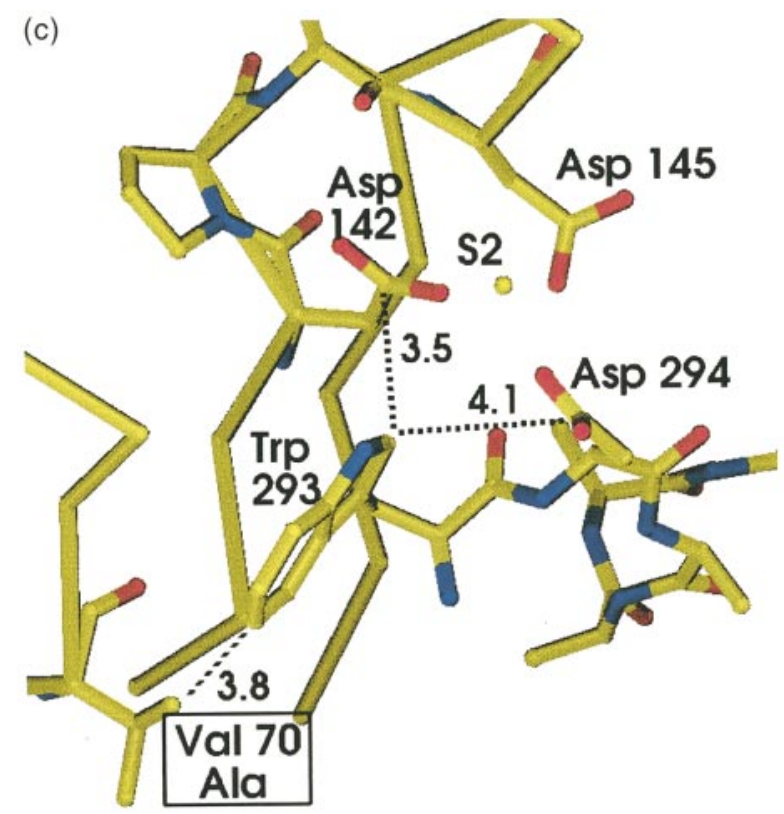

Figure 4. (a) A diagram showing the superposition of Hal2p (thick yellow $\mathrm{C}^{\alpha}$ trace) and human inositol monophosphatase (thin cyan $\mathrm{C}^{\alpha}$ trace); the Figure shows the hydrogen bonding network involving Asp145 and the salt bridge Arg152 Asp263, linking $\alpha 5$ and the loop between $\beta 9$ and $\alpha 7$. (b) A detail of this salt bridge and (c) a section of Hal2p structure showing where the point mutation V70A is located. Point mutations are highlighted, distances are in $\AA$.

to predict based on the crystallographic structure information (Table 2 and Figure 2(b) and (c)). Val70 is strictly conserved among Hal2p and the PAP phosphatases of Arabidopsis and it is located between the $\mathrm{N}$ and C-terminal domains. Val70 CG1 is making hydrophobic interactions with essential Trp293, which holds the carboxylates Asp142 and Asp294 at site 2 (Figure 4(c)). These carboxylates are directly involved in metal binding so, a subtle modification in their conformations would result in an altered specificity at this site, as is observed in the biochemical analysis. Another possible explanation is that this mutation affects the relative orientation of the $\mathrm{N}$ and $\mathrm{C}$-terminal domains. This type of change is likely to affect the geometry of the metal binding sites of the enzyme 

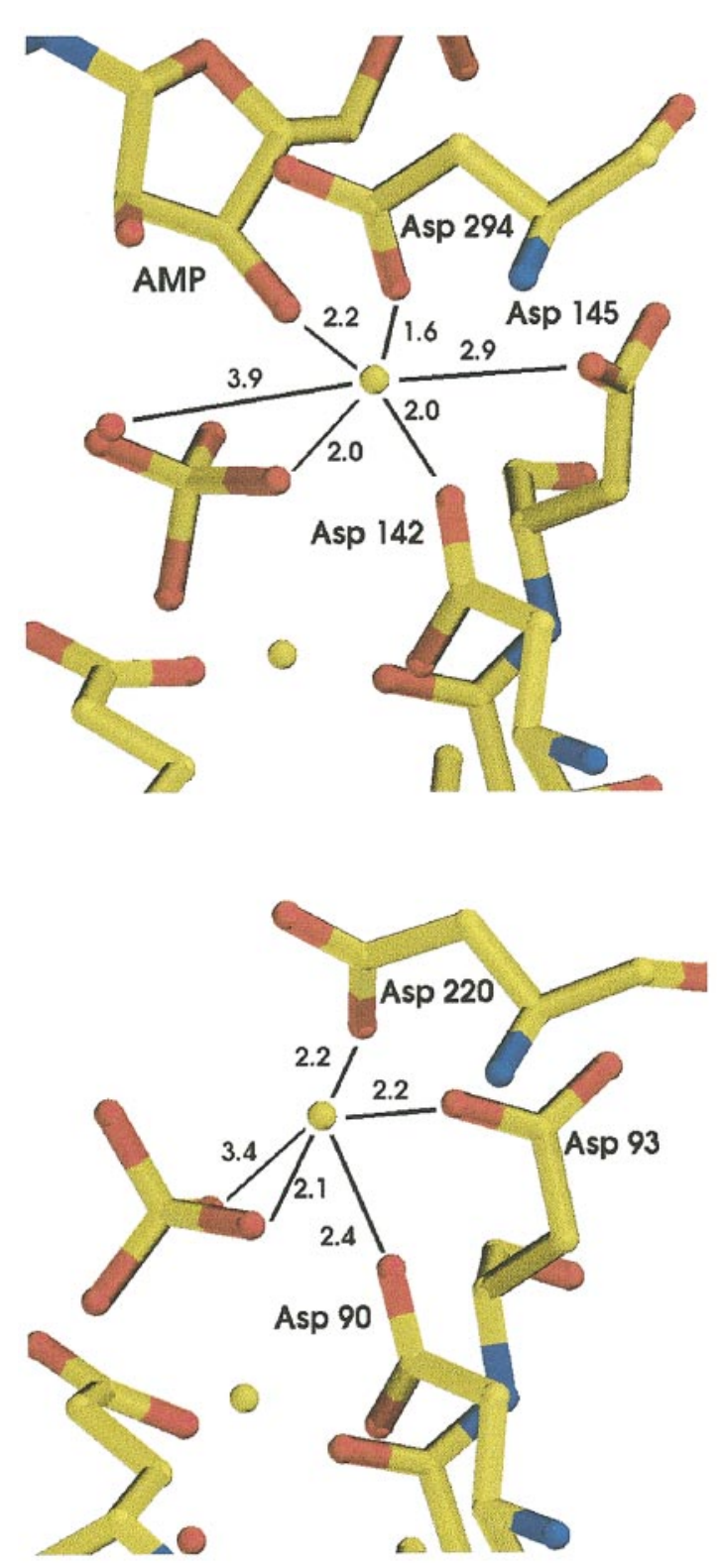

Figure 5. Comparison of metal binding site 2 in Hal2p (top) and in human inositol monophosphatase (bottom). Distances are in $\AA$.

since they are located at the interface between the two domains. This question could be answered by solving the crystal structure of this mutation and comparing the resulting changes in the architecture of the active site.

Given the similarity in the structures, we hypothesized that the information learned for Hal2p may be applicable to human IMPase. Analysis of the superimposed structures indicates that Glu238 of Hal2p has no structural analogue in human but that Val70 is equivalent to Ile68 of human IMPase. As predicted, substitution of Ile68 for alanine renders human IMP approximately tenfold more resistant to inhibition by lithium chloride in vitro (Figure 3 ). This phenotype is similar to that observed for the V70A mutation of Hal2p and demonstrates that the structural change induced by this amino acid substitution results in a similar modification in the binding characteristics of metal binding site 2 in both enzymes. By analogy to the situation in Hal2p, the effect of the I68A mutation must be mediated by the altered hydrophobic interaction with Trp219 affecting the position of active site Asp220. It is plausible that the three previously described mutations of HAPase affecting cation sensitivity (H217Q, C218A and W219F) (Gore et al., 1993; Rees-Milton et al., 1997) also act by displacing Asp220.

As a general conclusion we propose that the combination of structural and mutagenic analysis of Hal2p started here may contribute to the generation of salt-tolerant crops by the genetic engineering of sodium-tolerant PAP phosphatases.

\section{Materials and Methods}

\section{Yeast strains and vectors}

The S. cerevisiae strain used here was RS1051 (MATa leu2-3,112 ura3-251,328,372 hal2::URA3) (Glaser et al., 1993). For crystallographic studies, HAL2 was subcloned into the yeast multicopy vector pRS421, which carries a LEU2 marker. This vector is derived from Yep 351 (Hill et al., 1986) where an expression cassette, containing the promoter $(0.75 \mathrm{~kb})$ and terminator $(1.1 \mathrm{~kb})$ regions of the yeast PMA1 gene, has been introduced into the HindIII site. For the mutant screening, HAL2 was subcloned into a centromeric, LEU2-containing, shuttling vector, pUN100 (Ellege \& Davis, 1988).

\section{Hal2p purification from yeast}

For crystallographic studies, native Hal2p was purified by affinity chromatography using a PAP-agarose matrix (adenosine $3^{\prime} 5^{\prime}$-diphosphate agarose, SIGMA, ref. A3640). This method is based on that of Ramaswamy \& Jakoby (1987). The crude extract from strain RS1051[pRS421-HAL2] was prepared by resuspending collected cells in a buffer containing $1.2 \%(\mathrm{w} / \mathrm{v})$ sucrose, $10 \mathrm{mM}$ Tris$\mathrm{HCl}$ (pH 7.6), $20 \mathrm{mM} \mathrm{KC1,} 1 \mathrm{mM}$ EDTA, 0.2 mM DTT, $0.5 \mathrm{mM}$ PMSF, and $25 \mathrm{mg} \mathrm{ml}^{-1}$ of chimostatin. Following physical disruption of the cells, the soluble extract was made $1 \mathrm{mM}$ with respect to $\mathrm{MgCl}_{2}$ and $6 \mathrm{mM}$ with respect to $\mathrm{LiCl}$ and then loaded onto the column. The column was washed with buffer $1(1 \mathrm{mM} \mathrm{MgCl}, 6 \mathrm{mM}$ LiCl, $20 \mathrm{mM}$ Tris- $\mathrm{HCl}$ (pH 7.6), 5 mM 2-mercaptoethanol, $20 \%$ sucrose) and Hal2p eluted with buffer 2 (10 mM EDTA, $20 \mathrm{mM}$ Tris-HC1(pH 7.6), $5 \mathrm{mM}$ 2-mercaptoethanol, $20 \%$ sucrose). Hal2p was purified to homogeneity with an overall recovery of $1 \%$ (1 litre yeast culture gave $3 \mathrm{mg}$ ).

\section{Functional screening for lithium tolerant hal2 mutants}

A library of random HAL2 mutants was generated by introducing the pUN100-HAL2 vector into the E. coli XLI-Red strain $\left(m u t D^{-} ; m_{u t S}^{-} ; m_{t} T^{-}\right)$and preparing DNA as recommended by the manufacturer (Stratagene). The yeast strain RS1051 was transformed with the HAL2 random library and transformants were initially selected 
by leucine prototrophy in minimal medium $(0.7 \%(\mathrm{w} / \mathrm{v})$ yeast nitrogen base, $2 \%(\mathrm{w} / \mathrm{v})$ glucose and $50 \mathrm{mM}$ succinic acid, $\mathrm{pH}$ 5) supplemented with methionine $(100 \mu \mathrm{g}$ $\mathrm{ml}^{-1}$ ). Approximately $1.5 \times 10^{5}$ primary transformants were obtained and pooled together. In order to identify clones which both complement the methionine auxotrophy of the original hal2 strain and exhibit improved lithium tolerance, the primary transformants were spread on minimal media lacking methionine and containing $100 \mathrm{mM}$ lithium chloride. After secondary screening of 63 possible mutants, ten clones were subjected to plasmid rescue and phenotype confirmation upon retransformation into the original hal 2 strain.

\section{Generation of site-directed mutants of inositol monophosphatase}

Isoleucine 68 of human inositol monophosphatase (McAllister et al., 1992) was changed to alanine using an overlapping PCR approach. The primers used were as follows: $5^{\prime}$ external: 5'-ATTATA-GAGCTCATGGCTGA TCCTTGGCAGGAATGC-3'; 3' external: 5'-CGCTCG AGCGGTTAATCTTCGT-3'; 5' internal: 5'-AAGTATCCATCTCACAGTTTCGCTGGTGAA-3'; $3^{\prime}$ internal: $5^{\prime}$ AGCTGCCACAGATTCTTCACCAGCGAAACT-3'. The product of the second round PCR was digested with SacI and XhoI and subcloned into the pET28a vector (Novagen) to produce a histidine-tagged fusion protein. All constructs were confirmed by sequencing. (Restriction sites are in bold face, the start and stop codons are underlined, and the mutated base-pairs appear in italics.)

\section{Expression of Hal2p and mutants in E coli and protein purification}

As the purification procedure of native Hal2p by PAPagarose chromatography depends on inhibiting the enzymatic reaction with lithium, we purified the lithiumresistant HAL2 mutations as poly-histidine tagged fusion proteins. The open reading frames of Hal2p, human inositol monophosphatase and the mutant forms were amplified by PCR and subcloned into the SacI and HindII sites of the pET28a vector (Novagen). All constructs were confirmed by sequencing and the proteins were produced and purified from the E. coli strain BL21(DE3) using metal chelation chromatography, as recommended by the manufacturer. The proteins were visualized and quantified using SDS-PAGE.

\section{Phosphatase enzymatic assay}

Phosphatase activity was assayed by quantifying the inorganic phosphate liberated in each reaction using the malachite green procedure (Baykov et al., 1988) as previously described by Murguia et al. (1995).

\section{Crystallization, data collection, structure solution and refinement}

Crystallization experiments were carried out in the presence of the natural substrate and the metal cofactors required for catalysis and inhibition. Protein was concentrated to $5 \mathrm{mg} \mathrm{ml}^{-1}$ and made $5 \mathrm{mM} \mathrm{MgCl}_{2}$, $0.6 \mathrm{mM} \mathrm{PAP}$ and $5 \mathrm{mM} \mathrm{Li} \mathrm{SO}_{4}$. Experiments were carried out at room temperature. Crystals were grown by vapour diffusion from drops containing Hal2p and reservoir $(30 \%(\mathrm{w} / \mathrm{v})$ PEG5000 MME, $0.1 \mathrm{M}$ sodium acetate, $5 \mathrm{mM}$ beta-mercaptoethanol, $0.1 \mathrm{M}$ Mes $\mathrm{pH}$ 6.5) solutions in a 1:1 ratio. X-ray data from native and derivatives were collected using $\mathrm{CuK}_{\alpha}$ radiation on an in-house source at room temperature (Mar 345 and $\mathrm{R}$ axis 4 image plate detectors on Enraf Nonius and Rigaku rotating anode generators, respectively). Native crystals were also frozen to collect a higher resolution data set to be used for refinement. The data were processed using MOSFLM (Leslie, 1987) in space group $P 2_{1}$. The coordinates of one site from the lead acetate derivative were determined using the Patterson interpretation routine of SHELX (Sheldrick, 1991). All the other sites were obtained by difference Fourier techniques and by cross-phasing with the known lead phases. All calculations were performed using programs of the CCP4 package (Bailey, 1994) and the final refinement and solvent flattering were performed using SHARP (de la Fortelle \& Bricogne, 1997). Despite the good phasing statistics after refinement of the heavy atom positions, some areas of the solvent flattened map were uninterpretable. This was due to the similar positions of lead, platinum, gadolinium and samariurn heavy atoms. All were bound to the magnesium sites. This caused an overestimation in the figures of merit and an underestimation of the phase error. Following Chabriere et al. (1999) we used the lead derivative as native and the native as one derivative. This improved the scaling process, so that it was easier to find minor heavy atom positions, and estimate the phase errors. In this way the phasing statistics were slightly worse for the derivatives sharing heavy atom sites but probably more meaningful. Using both the conventional experimental and the map calculated following Chabriere et al. (1999), we were able to build a model, using $\mathrm{O}$ (Jones et al., 1991) and refine it using the torsion dynamics routine of XPLOR (Brunger, 1988) at $2.5 \AA$ resolution. Several cycles of restrained refinement at high resolution using REFMAC (Murshudov et al., 1997), followed by interactive model building were carried out (see Table 1). The stereochemistry of the model was verified with PROCHECK (Laskowski et al., 1993). Ribbon figures were produced using MOLSCRIPT (Kraulis, 1991) and RASTER (Merrit \& Murphy, 1994). Energy minimization of the AMP molecule was performed using CERIUS2 default force fields and excluding those terms corresponding to bond distances and angles.

\section{PDB accession codes}

The coordinates and structure factor amplitudes are deposited in the Protein Data Bank (PDB code: 1QGX).

\section{Acknowledgements}

We thank Dr C. Abell and Professor D. Gani for critical comments on the manuscript and Drs C.I. Ragan and G. McAllister (Merck Sharp \& Dohme Research Laboratories) for the human IMPase cDNA. A.A. is a holder of a contract of the Spanish Ministerio de Eduacion y Cultura and he was funded in part by a ROPA grant from the BBSRC and by a grant of the Spanish "Plan Nacional de Biotecnologia" (CICYT, Madrid). S.P. is supported by a BBSRC earmarked studentship. T.L.B. thanks the Wellcome Trust for provision of $\mathrm{X}$-ray and computing equip- 
ment. Work in R.S. laboratory was funded by grants of the Spanish "Plan Nacional de Biotecnologia" (CICYT, Madrid) and the European Programme on Biotechnology (European Commission, Brussels). L.Y. holds an International Research Fellow Award of the National Science Foundation (USA) and P.L.R. is a holder of a contract of the Spanish Ministerio de Eduacion y Cultura.

\section{References}

Allen, F. H., Davies, J. E., Galloy, J. J., Johnson, O., Kennard, O., Macrae, C. F., Mitchell, E. M., Mitchell, G. F., Smith, J. M. \& Watson, D. G. (1991). The development of version-3 and version- 4 of the Cambridge structural database system. J. Chem. Info. Comp. Sci. 31, 187-204.

Arrillaga, I., Gil-Mascarell, R., Gisbert, C., Sales, E., Montesinos, C., Serrano, R. \& Moreno, V. (1998). Expression of the yeast HAL2 gene in tomato increases the in vitro salt tolerance of transgenic progenies. Plant Sci. 136, 219-226.

Bailey, S. (1994). The CCP4 suite - programs for protein crystallography. Acta Crystallog. sect. D, 50, 760-763.

Baykov, A. A., Evtushenko, O. A. \& Avaeva, S. M. (1988). A malachite green procedure for orthophosphate determination and its use in alkaline phosphatase-based enzyme immunoassay. Anal. Biochem. 171, 266-270.

Bone, R., Springer, J. P. \& Atack, J. R. (1992). Structure of inositol monophosphatase, the putative target of lithium-therapy. Proc. Natl Acad. Sci. USA, 89, 10031-10035.

Bone, R., Frank, L., Springer, J. P., Pollack, S. J., Osborne, S., Atack, J. R., Knowles, M. R., Mcallister, G., Ragan, C. I., Broughton, H. B., Baker, R. \& Fletcher, S. R. (1994a). Structural analysis of inositol monophosphatase complexes with substrates. Biochemistry, 33, 9460-9467.

Bone, R., Frank, L., Springer, J. P. \& Atack, J. R. (1994b). Structural studies of metal-binding by inositol monophosphatase - evidence for 2-metal ion catalysis. Biochemistry, 33, 9468-9476.

Brunger, A. T. (1988). Crystallographic refinement by simulated annealing application to a $2.8 \AA$ resolution structure of aspartate-aminotransferase. J. Mol. Biol. 203, 803-816.

Chabriere, E., Charon, M. H. \& Vellieux, F. M. D. (1999). PBR: a heavy-atom refinement and phasing procedure to reduce phase bias when heavy-atom derivatives contain common sites. Acta Crystallog. sect. $D, 55,469-472$.

Cole, A. G., Wilkie, J. \& Gani, D. (1995). Probes for the position and mechanistic role of the 2nd catalytic magnesium-ion in the inositol monophosphatase reaction. J. Chem. Soc. Perkin Trans. 1, 21, 2695-2707.

Dichtl, B., Stevens, A. \& Tollervey, D. (1997). Lithium toxicity in yeast is due to the inhibition of RNA processing enzymes. EMBO J. 16, 7184-7195.

Ellege, S. J. \& Davis, R. W. (1988). A family of versatile centromeric vectors designed for use in the sectoring-shuffle mutagenesis assay in Saccharomyces cerevisiae. Gene, 70, 303-312.

de la Fortelle, E. \& Bricogne, G. (1997). Maximum-likelihood heavy-atom parameter refinement for multiple isomorphous replacement and multiwavelength anomalous diffraction methods. Methods Enzymol. 276, $472-494$.
Gil-Mascarell, R., Lopez-Coronado, J. M., Belles, J. M., Serrano, R. \& Rodriguez, P. L. (1999). The Arabidopsis HAL2-like gene family includes a novel sodiumsensitive phosphatase. Plant J. 17, 373-383.

Glaser, H. U., Thomas, D., Gaxiola, R., Montrichard, F., Surdun-Kerjan, Y. \& Serrano, R. (1993). Salt tolerance and methionine biosynthesis in Saccharomyces cerevisiae involve a putative phosphatase gene. EMBO J. 12, 3105-3110.

Glusker, J. P. (1991). Structural aspects of Metal liganding to functional groups in proteins. In Metaloproteins: Structural Aspects, (Anfinsen, C. B., Edsal, J. E., Richards, M. R. \& Eisenberg, D. S., eds), Advan. Protein Chem. vol. 42, pp. 1-76, .

Goldman, A. (1995). How to make my blood boil. Structure, 3, 1277-1279.

Gore, M. G., Greasley, P., Mcallister, G. \& Ragan, C. (1993). Mammalian inositol monophosphatase - the identification of residues important for the binding of $\mathrm{Mg}^{2+}$ and $\mathrm{Li}^{+}$ions using fluorescence spectroscopy and site-directed mutagenesis. Biochem. J. 296, 811-815.

Hill, J. E., Myers, A. M., Koerner, T. J. \& Tzagoloff, A. (1986). Yeast/E. coli shuttle vectors with multiple unique restriction sites. Yeast, 2, 163-167.

Jones, T. A., Zou, J. Y., Cowan, S. W. \& Kjeldgaard, M. (1991). Improved methods for model building in electron density maps and the location of errors in these models. Acta Crystallog. sect, A, 47, 110-119.

Kraulis, P. J. (1991). MOLSCRIPT - A program to produce both detailed and schematic plots of protein structures. J. Appl. Crystallog. 24, 946-950.

Laskowski, R. A., Macarthur, M. W., Moss, D. S. \& Thornton, J. M. (1993). PROCHECK - A program to check the stereochemical quality of protein structures. J. Appl. Crystallog. 26, 283-291.

Leslie, A. G. W. (1987). MOSFLM. In Proceedings of the CCP4 Study (Weekend, J. R., Machin, P. A. \& Papiz, M. Z., eds), pp. 39-50, SERC Daresbury Lab., Warrinton, UK.

Lopez, F., Leube, M., Gil-Mascarell, R., Navarro-Avino, J. P. \& Serrano, R. (1999). The yeast inositol monophosphatase is a lithium- and sodium-sensitive enzyme encoded by a non-essential gene pair. Mol. Microbiol. 31, 1255-1264.

Lopez-Coronado, J. M., Lesage, F., Belles, J. M., Serrano, R. \& Rodriguez, P. L. (1999). A novel mammalian lithium-sensitive enzyme with a dual enzymatic activity, 3'-phosphoadenosine 5'-phosphate phosphatase and inositol-polyphosphate 1-phosphatase. J. Biol. Chem. 274, 16034-16039.

McAllister, G., Whiting, P., Hammond, E. A., Knowls, M. R., Atack, J. R., Bailey, F. J., Maigetter, R. \& Ragan, C. I. (1992). cDNA cloning of human and rat brain myo-inositol monophosphatase. Biochem. J. 284, 749-754.

Merritt, E. A. \& Murphy, M. E. P. (1994). RASTER3D Version-2.0 - a program for photorealistic molecular graphics. Acta Crystallog. sect. D, 50, 869-873.

Murguia, J. R., Belles, J. M. \& Serrano, R. (1995). A salt-sensitive $3^{\prime}\left(2^{\prime}\right), 5^{\prime}$-bisphosphate nucleotidase involved in sulfate activation. Science, 267, 232-234.

Murguia, J. R., Belles, J. M. \& Serrano, R. (1996). The yeast HAL2 nucleotidase is an in vivo target of salt toxicity. J. Biol. Chem. 271, 29029-29033.

Murshudov, G. N., Vagin, A. A. \& Dodson, E. J. (1997). Refinement of macromolecular structures by the maximum-likelihood method. Acta Crystallog. sect. $D, 53,240-255$. 
Nahorski, S. R., Ragan, C. I. \& Challiss, R. A. J. (1991). Lithium and the phosphoinositide cycle: an example of uncompetitive inhibition and its physiological consequences. Trends Pharmacol. Sci. 12, 297-303.

Peng, Z. \& Verma, D. P. S. (1995). A rice HAL2-like gene encodes a $\mathrm{Ca}^{2+}$-sensitive $3^{\prime}\left(2^{\prime}\right), 5^{\prime}$-diphosphonucleoside $3^{\prime}\left(2^{\prime}\right)$ phosphohydrolase and complements yeast met22 and Escherichia coli cysQ mutations. J. Biol. Chem. 270, 29105-29110.

Pollack, S. J., Knowles, R., Broughton, H. B., Ragan, C. I., Osborne, S. A. \& McAllister, G. (1993). Probing the role of metal-ions in the mechanism of inositol monophosphatase by site-directed mutagenesis. Eur. J. Biochem. 217, 281-287.

Quintero, F. J., Garciadeblas, B. \& Rodriguez-Navarro, A. (1996). The SALI gene of Arabidopsis, encoding an enzyme with $3^{\prime}\left(2^{\prime}\right), 5^{\prime}$-bisphosphate nucleotidase and inositol polyphosphate 1-phosphatase activities, increases salt tolerance in yeast. Plant Cell, 8, 529537.

Ramaswamy, S. G. \& Jakoby, W. B. (1987). (2') 3',5Bisphosphate nucleotidase. J. Biol. Chem. 262, 1004410047.

Rees-Milton, K., Thorne, M., Greasley, P., Churchich, J. \& Gore, M. G. (1997). Detection of metal binding to bovine inositol monophosphatase by changes in the near and far ultraviolet regions of the CD spectrum. Eur. J Biochem. 246, 211-217.

Roth, J. A., Rivett, A. J. \& Renskers, K. J. (1982). Sulfate Metabolism and Sulfate Conjugation (Mulder, G. J., Caldwell, J., Van Kempen, G. M. J. \& Vonk, R. J., eds), pp. 107-114, Taylor and Francis, London, UK.

Serrano, R. (1996). Salt tolerance in plants and microorganisms: toxicity targets and defense responses. Int. Rev. Cytol. 165, 1-52.
Sheldrick, G. M. (1991). The SHELXS system. In Crystallographic Computing (Podjarny, P. D. \& Thiery, J. D., eds), vol. 5, pp. 145-159, IUCR Oxford University Press, Oxford, UK.

Strater, N., Lipscomb, W. N., Klabunde, T. \& Krebs, B. (1996). Two-metal ion catalysis in enzymatic acyland phosphoryl-transfer reactions. Angew. Chem. Int. Ed. Eng. 35, 2024-2055.

Villeret, V., Huang, S. H., Zhang, Y. P., Xue, Y. F. \& Lipscomb, W. N. (1995). Crystal-structure of spinach chloroplast fructose-1,6-bisphosphatase at 2.8 angstrom resolution. Biochemistry, 34, 4299-4306.

Wilke, J., Cole, A. G. \& Gani, D. (1995). 3-Dimensional interactions between inositol monophosphatase and its substrates, inhibitors and metal-ion cofactors. J. Chem. Soc. Perkin Trans. 1, 21, 27092727.

Xue, Y. F., Huang, S. H., Liang, J. Y., Zhang, Y. P. \& Lipscomb, W. N. (1994). Crystal-structure of fructose-1,6-bisphosphatase complexed with fructose 2,6-bisphosphate, AMP, and $\mathrm{Zn}^{2+}$ at 2.0-Ångstrom resolution - aspects of synergism between inhibitors. Proc. Natl Acad. Sci. USA, 91, 1248212486.

York, J. D., Ponder, J. W., Chen, Z. W., Matthews, F. S. \& Majerus, P. W. (1994). Crystal structure of inositol polyphosphate 1-phosphatase at 2.3-Ångstrom resolution. Biochemistry, 33, 13164-13171.

York, J. D., Ponder, J. W. \& Majerus, P. W. (1995). Definition of a metal-dependent $\mathrm{Li}^{+}$-inhibited phosphomonoesterase protein family based upon a conserved 3-dimensional core structure. Proc. Natl Acad. Sci. USA, 92, 5149-5153.

Edited by R. Huber

(Received 6 September 1999; received in revised form 15 November 1999; accepted 20 November 1999) 